Patricia N. Ayson, MD', Jan Eero G. Lopez, MD', Erasmo Gonzalo DV Llanes, MD 1,3

'Department of Otolaryngology Head and Neck Surgery Quirino Memorial Medical Center

2Departments of Otolaryngology Head and Neck Surgery

St Luke's Medical Center and The Medical City ${ }^{3}$ Department of Otorhinolaryngology College of Medicine - Philippine General Hospital University of the Philippines Manila
Correspondence: Patricia N. Ayson, MD Quirino Memorial Medical Center Katipunan Ave., Project 4, Quezon City 1105 Philippines

Phone: (632) 4212250 local 117/129

Fax: (632) 9134758

Email: patty_tlp@yahoo.com

Reprints will not be available from the author.

No funding support was received for this study. The authors signed a disclosure that they have no proprietary or financial interest with any organization that may have a direct interest in the subject matter of this manuscript, or in any product used or cited in this study.

Presented at:

1. North East Manila ENT Consortium Research Contest (3rd Place) September 2005

2. Descriptive Research Contest (2nd Place), Philippine Society of Otolaryngology Head and Neck Surgery 49th Annual Convention, Westin Philippine Plaza Hotel, Manila, December 1, 2005

\section{Chronic Suppurative Otitis Media: Bacteriology and Drug Sensitivity Patterns at the Quirino Memorial Medical Center (2004-2005): A Preliminary Study}

\begin{abstract}
Objectives: To identify the bacterial pathogens associated with chronic suppurative otitis media and their antimicrobial sensitivity and resistance.

Study Design: Cross-sectional survey.

Setting: The study was carried out from July 2004-July 2005 at the outpatient clinic of a government tertiary hospital.

Number of subjects: A total of 32 patients (54 ears) with unilateral or bilateral active chronic suppurative otitis media.

Results: Bacterial pathogens of 54 ear discharge samples from CSOM were studied. Among them, $42(78 \%)$ were pure cultures and $9(16.7 \%)$ were mixed, only $3(5.6 \%)$ of the submitted samples had no growth. There were 42 pure isolates, the most common of which was Staphylococcus aureus 21 (50\%), followed by Pseudomonas aeruginosa 14 (33.3\%). Drug sensitivity pattern of Staphylococcus aureus showed that $61.9 \%$ were resistant to Penicillin while more than $90 \%$ were sensitive to Aminoglycosides and Clindamycin. Pseudomonas aeruginosa was resistant to penicillin in $64.3 \%$ of cases and Ciprofloxacin was active against pseudomonas in $85.7 \%$.
\end{abstract}

Conclusion: The most common bacterial pathogens from CSOM include Staphylococcus aureus and Pseudomonas aeruginosa. Majority of the isolates of Staphylococcus aureus were resistant to penicillin. Aminoglycosides, macrolides and quinolones were effective against most of the isolates of Staphylococcus aureus.

Keywords: chronic otitis media, perforated tympanic membrane, chronic aural discharge

CHRONIC suppurative otitis media (CSOM), also called chronic active mucosal otitis media, chronic oto-mastoiditis, and chronic tympanomastoiditis, is a chronic inflammation (>6-12 weeks) of the middle ear and mastoid cavity, which presents with recurrent ear discharge or otorrhea through a perforated tympanic membrane'. These perforations may arise traumatically, iatrogenically with tube placement, or after an episode of acute otitis media (AOM), which decompresses through a tympanic perforation ${ }^{2}$. CSOM is one of the most important causes of preventable hearing loss in the Philippines and other developing countries'.

Previous studies have provided evidence for the resolution of CSOM using otic drops alone as the first line of treatment ${ }^{4,5}$. This is because of their good penetration and better availability through the perforation compared to systemic antibiotics. Other studies, however, showed an increasing number of treatment failures ${ }^{6}$. Treatment for CSOM varies considerably based on the 
ORIGINAL ARTICLES

Table 1. Inclusion and exclusion criteria for selecting patients in the study

Criteria for selection of patients for inclusion in the study

Patients presenting with chronic ear discharge for more than $6-12$ weeks

Patients with perforated tympanic membrane

Patients must not have received ototopical or systemic antibiotics $(>12 \mathrm{wks})$ prior to the inclusion dates set before the study was started

\begin{tabular}{|c|c|c|c|}
\hline Cultures & Isolated bacterial pathogens & Total & \\
\hline \multirow[t]{6}{*}{ Pure } & & & $\%$ \\
\hline & Staphylococcus aureus & 21 & 50.0 \\
\hline & Pseudomonas aeruginosa & 14 & 33.3 \\
\hline & Streptococcus viridans & 3 & 7.1 \\
\hline & Proteus mirabilis & 4 & 9.5 \\
\hline & Total : & 42 & $(77.8 \%)$ \\
\hline \multirow[t]{5}{*}{ Mixed } & & & $\%$ \\
\hline & $\begin{array}{l}\text { Staphylococcus aureus and } \\
\text { proteus mirabilis }\end{array}$ & & \\
\hline & $\begin{array}{l}\text { Staphylococcus aureus and } \\
\text { Klebsiella sp. }\end{array}$ & 3 & 33.3 \\
\hline & $\begin{array}{l}\text { Pseudomonas aeruginosa and } \\
\text { staph. epidermidis }\end{array}$ & 1 & 11.1 \\
\hline & Total: & 9 & $(16 / 7 \%)$ \\
\hline No growth & & 3 & $(5.6 \%)$ \\
\hline Total & & 54 & $(100 \%)$ \\
\hline
\end{tabular}

duration of symptoms, past therapeutic failures, severity of current symptoms and culture and sensitivity profile. Patients diagnosed with CSOM warranting systemic antimicrobial therapy previously needed discharge cultures to determine bacterial profiles for the selection of systemic therapy, until studies identified the common bacterial pathogens serving as bases for the selection of systemic antimicrobials for empiric therapy. However, morbidity from this disease remains significant in our country despite the use of systemic antibiotics to treat the illness and its complications ${ }^{1}$, with antibiotic resistance becoming a serious and major issue ${ }^{7}$. According to Wintermeyer, et $\mathrm{al}^{6}$, and Aslam, et $\mathrm{al}^{8}$, treatment of CSOM with otic, oral or parenteral drugs should be guided by ear fluid culture results. Individual data on culture studies could suggest choices for instituting the narrowest spectrum systemic antibiotics applicable in our setting, significantly assuring a most efficient and cost-effective protocol of treatment. However, this may be too expensive for most of our patients, and much of the morbidity of CSOM comes from treatment failure.

Recognizing the need for awareness of the current pattern of bacterial pathogens isolated from CSOM in our setting, the objective of our study is to identify the common bacterial isolates from patients diagnosed with chronic suppurative otitis media at the Quirino Memorial Medical Center and to determine the sensitivity and resistance of these bacterial isolates to commonly used antimicrobials.

\section{METHODOLOGY}

All patients diagnosed with chronic suppurative otitis media (using theWorld Health Organization criteria for CSOM, 2004)' at the outpatient clinic of the Quirino Memorial Medical Center from July 2004 to July 2005 and from whom informed consent was obtained, (signed by the patient or parent/guardian) were considered for inclusion.

A total of 32 patients with unilateral or bilateral active CSOM satisfying the inclusion criteria set before the study (Table 1), were assessed at our outpatient clinic. A diagnosis of CSOM was made using the WHO definition (2004): 1) a history of chronic purulent ear discharge (>6-12 weeks), and 2) tympanic perforation, verified with otoscopic examination. Removal of cerumen and suctioning of ear discharge were done to adequately visualize the character of the discharge, tympanic membrane perforation, and status of the middle ear (polypoid, thickened, eroded). The evaluation and diagnosis was exclusively made by an otorhinolaryngologist before inclusion of the patient.

Aseptic microsurgical techniques were observed in the collection of specimens. 1) Each tympanic membrane was adequately visualized 2) a sterile cotton pledget soaked in povidone-iodine (Betadine ${ }^{T M}$ ) was swabbed 3 times around the external auditory canal (EAC) 3) a sterile cotton swab soaked in $70 \%$ ethyl alcohol was likewise applied thrice around the EAC 4) a sterile, dry micro-cotton swab was applied to the fluid draining from the tympanic membrane (TM) avoiding contact with the EAC walls. Each specimen bottle was labeled with the patient's name and ear laterality and submitted for direct culture for aerobes and sensitivity studies at the Department of Laboratories within 15 minutes of collection.

Direct culture material was seeded onto sheep blood agar and chocolate agar plates, incubated in the presence of oxygen for 24 hours at $37^{\circ} \mathrm{C}$ and subjected to automated bacterial identification. Isolates yielding pure cultures were further studied for antimicrobial sensitivity and resistance, using drugs chosen from commonlyprescribed medications for patients with CSOM in our institution. This study was limited to identification of aerobic bacterial isolates from the samples submitted for culture. No studies were done for anaerobes, viruses or fungi. This study adhered to the Declaration of Helsinki and was approved by the Research and Ethics Committee of the Residents Training Program of our Institution. 


\section{RESULTS}

Fifty four specimens were obtained from the 32 patients satisfying our inclusion criteria. On the average, patients were 19-20 years old \pm 13-14, ranging from 7 to 41 years old. Half of the patients (16/32) were pediatric patients, and half were adults. The proportion of males were larger $(59.37 \%$ or $19 / 32)$ than females $(40.62 \%$ or $13 / 32)$, with a ratio of 1.46:1.

Fifty one of the 54 samples or $94.4 \%$ grew bacterial isolates. Forty two of the samples sent for direct culture yielded pure cultures (77.8\%), 9 were mixed cultures (16.7\%) and 3 samples (5.6\%) had no growth (Table 2).

Direct cultures revealed pathogens in 51 (94.4\%) of 54 samples. Major bacteria isolated from pure cultures were Staphylococcus aureus in 50\% (21/42), Pseudomonas aeruginosa in 33.3\% (14/42) Streptococcus viridans $7.1 \%(3 / 42)$ and proteus mirabilis $9.5 \%(4 / 42)$. The most common pediatric age-group isolates were Staphylococcus aureus $(76.19 \%$ or 16 121) and Pseudomonas aeroginosa (78.57\% or 11/14).

Most mixed cultures $88.89 \%$ (8/9) were isolated from the adult group. Combinations included Staphylococcus aureus and Proteus mirabilis in 55.6\% (5/9), Staphylococcus aureus and Klebsiella spp. in 3 (33.3\%) and Pseudomonas aeruginosa with Staphylococcus epidermidis in 1 (11.1\%) (Table 3).

Drug sensitivity patterns for pure cultures (Table 4 ) revealed $61.9 \%$ of isolated Staphylococcus aureus were resistant to penicillin (13/21), with only $38 \%$ sensitive to the drug. Pseudomonas aeruginosa (Table 5 ) showed $64.3 \%$ resistance to penicillin (all in the pediatric group) while Ciprofloxacin was active against $85.7 \%$ (12/14) of isolates followed by amikacin (83.3\%) and gentamicin (50\%). Pseudomonas was resistant to macrolides in $77.8 \%$. All isolates of Streptococcus viridans and Staphylococcus mirabilis tested for penicillin revealed $100 \%$ sensitivity to the drug (Table 6).

\section{DISCUSSION}

The average incidence of chronic suppurative otitis media at the Quirino Memorial Medical Center (based on retrospective data reviewed from 2000 to 2003) is 34 cases per year. Several surveys among children in Metro Manila conducted by Yabut (1994, in PSOHNS $2004^{9}$ ) estimated the prevalence of CSOM at $2.5-29.4 \%$. The prevalence of CSOM in various African countries is reported between 2 and $6 \%$, whereas it is below $1 \%{ }^{5}$ in the USA and Europe. The estimated yearly incidence of CSOM in developing countries (including the Philippines) is 39. ${ }^{3}$ According to World Health Organization (WHO) data, a prevalence of $4 \%$ is representative of Asian countries, obviously a lot more than rate patterns in developed countries. There is no gender predilection. Although most surveys and studies on CSOM were done among children, and most developing countries have predominantly young populations in whom CSOM is most prevalent, CSOM can affect both pediatric and adult groups, as illustrated in this study.

The Philippines has been classified by the WHO as belonging to the high prevalence group $(2-4 \%)^{2}$ of CSOM, and this avoidable burden of disease must be addressed. Systemic antimicrobial agents may be necessary for the treatment of CSOM and the type of antimicrobial and
Table 3. Bacteriology of ear discharge specimens according to age group $(n=51)$

\begin{tabular}{|c|c|c|c|c|c|c|}
\hline \multirow{3}{*}{$\begin{array}{l}\text { Culture } \\
\text { Pure }\end{array}$} & \multicolumn{4}{|c|}{ Age group } & \multicolumn{2}{|c|}{ Total } \\
\hline & \multicolumn{2}{|c|}{ pediatric } & \multicolumn{2}{|c|}{ adult } & \multirow[b]{2}{*}{ No. } & \multirow{2}{*}{$\begin{array}{l}\% \\
\%\end{array}$} \\
\hline & No. & $\%$ & No. & $\%$ & & \\
\hline Staphylococcus aureus & 16 & 76.2 & 5 & 23.8 & 21 & 38.9 \\
\hline Pseudomonas aeruginosa & 11 & 78.6 & 3 & 21.4 & 14 & 25.9 \\
\hline Streptococcus viridans & 0 & 0 & 3 & 100 & 3 & 5.6 \\
\hline Proteus mirabilis & 3 & 75.0 & 1 & 25.0 & 4 & 7.4 \\
\hline $\begin{array}{l}\text { Staphylococcus aureus and } \\
\text { Proteus mirabilis }\end{array}$ & 1 & 20 & 4 & 80.0 & 5 & 9.3 \\
\hline $\begin{array}{l}\text { Staphylococcus aureus } \\
\text { andKlebsiella spp. }\end{array}$ & 0 & 0 & 3 & 100 & 3 & 5.6 \\
\hline $\begin{array}{l}\text { Pseudomonas aeruginosa and } \\
\text { Staphylococcus epidermidis }\end{array}$ & 0 & 0 & 1 & 100 & 1 & 1.8 \\
\hline No growth & 2 & 66.7 & 1 & 33.3 & 3 & 5.6 \\
\hline
\end{tabular}

Table 4. Sensitivity studies for pure cultures (Staphylococcus aureus)

\begin{tabular}{|c|c|c|c|c|}
\hline & \multicolumn{4}{|l|}{ Culture } \\
\hline \multirow[t]{2}{*}{ Group of antibiotics } & \multicolumn{4}{|c|}{$\begin{array}{l}\text { Staphylococcus } \\
\text { aureus }\end{array}$} \\
\hline & Sensitive & $\%$ & Resistant & $\%$ \\
\hline \multicolumn{5}{|l|}{ Penicillins } \\
\hline Pen G & 8 & 38.1 & 13 & 61.9 \\
\hline Ampicillin & 8 & 38.1 & 13 & 61.9 \\
\hline Oxacillin & 6 & 33.3 & 12 & 66.7 \\
\hline \multicolumn{5}{|l|}{ Cephalosporins } \\
\hline Aminoglycosides & 18 & 94.7 & 1 & 5.3 \\
\hline \multicolumn{5}{|l|}{ Amikacin Gentamicin } \\
\hline Macrolides & 19 & 90.5 & 2 & 9.5 \\
\hline Chloramphenicol & 18 & 85.7 & 3 & 14.3 \\
\hline
\end{tabular}

Table 5. Sensitivity studies for pure cultures (Pseudomonas aeruginosa)

\begin{tabular}{|c|c|c|c|c|}
\hline \multirow{2}{*}{ Group of antibiotics } & \multicolumn{4}{|l|}{ Culture } \\
\hline & \multicolumn{4}{|c|}{$\begin{array}{l}\text { Pseudomonas } \\
\text { aeruginosa }\end{array}$} \\
\hline & Sensitive & $\%$ & Resistant & $\%$ \\
\hline $\begin{array}{l}\text { Penicillins Pen G } \\
\text { Ampicillin Oxacillin } \\
\text { Cephalosporins }\end{array}$ & 5 & 35.7 & $\begin{array}{l}9 \text { (all from } \\
\text { pediatric } \\
\text { group) }\end{array}$ & 64.3 \\
\hline Aminoglycosides & 107 & 83.3 & 27 & 16.7 \\
\hline Amikacin Gentamicin & & 50.0 & & 50.0 \\
\hline Macrolides & 2 & 22.2 & 7 & 77.8 \\
\hline \multicolumn{5}{|l|}{ Chloramphenicol } \\
\hline Quinolone (Ciprofloxacin) & 12 & 85.7 & 2 & 14.3 \\
\hline
\end{tabular}


Table 6. Sensitivity studies for pure cultures (Streptococcus viridans and Stapylococcus mirabilis)

\begin{tabular}{l|l|l|l|l|l|l|l|l|}
\hline Culture \\
Group of antibiotics & \multicolumn{3}{|c|}{ Streptococcus viridans } & \multicolumn{3}{|c|}{ Staphylococcus mirabilis } \\
& S & $\%$ & R & $\%$ & S & $\%$ & R & $\%$ \\
Penicillins & & & & & & & & \\
Pen G & 3 & 100 & 0 & & 4 & 100 & 0 & \\
Ampicillin & 3 & 100 & 0 & & 4 & 100 & 0 & \\
Oxacillin & 3 & 100 & 0 & & 4 & 100 & 0 & \\
Cephalosporins & & & & & 3 & 75.0 & 1 & 25.0 \\
Aminoglycosides & & & & & 4 & 100 & 0 & \\
Amikacin Gentamicin & & & & & & & & \\
Macrolides & & & & & 4 & 100 & 0 & \\
Chloramphenicol & & & & & 3 & 75.0 & 1 & 25.0 \\
Quinolone (Ciprofloxacin) & & & & & & & & \\
\hline
\end{tabular}

route of administration should be selected to suit the specific infecting organism(s). Over the years, research on common bacterial pathogens in CSOM has helped address and control the threat of its complications. Local bacteriological studies have been delimited by practical and procedural difficulties in, and inherent costs of obtaining outpatient middle ear discharge cultures.

Although the type of bacteria isolated in our study (Staphylococcus aureus, Pseudomonas aeruginosa, Proteus species, and polymicrobial isolates ${ }^{3}$ ), are similar to those previously reported ${ }^{3,5,8}$ the higher prevalence of Staphyloccoccus aureus, followed by Pseudomonas aeruginosa and mixed cultures differs from recent studies $3,5,6,7$ The individual percentage rates for $S$. aureus (50\%), P. aeruginosa and mixed cultures, also varied from those recently observed by Gupta, et al ${ }^{3}$ and the WHO where $P$. aeruginosa predominated in $48-98 \%$ of cases.

The presence of Streptococcus viridans and mixed cultures with Klebsiella spp. among the predominant pathogens (aside from S. aureus, $P$. mirabilis and $P$. aeruginosa) may generate varied hypotheses regarding the complex relationship between pathogen and host in the middle ear, which cannot be detected by traditional culture techniques.

Although the small sample size ( 42 isolates) may restrict the value of our findings, penicillin resistance of S. aureus in $61.9 \%$ of the cases and pseudomonas aeruginosa in $64.3 \%$, may suggest increasing penicillin resistance among pathogens that commonly cause CSOM. Penicillinresistant $P$. aeruginosa are much more common in children, and have become a major cause of therapeutic failure ${ }^{8,10}$. The increase in rates of penicillin-resistant $S$. aureus has been a hindrance to empirical treatment, necessitating reassessment of antibacterials of choice, and awakening interest in microbiological monitoring.

Chronic suppurative otitis media still ranks among the most important causes of preventable hearing loss. Morbidity from this disease remains significant in our country despite frequent use of systemic antibiotics to treat the illness and its complications. Concern for antimicrobial resistance with the widespread use of systemic antimicrobials is increasing, and it has been recommended that culture and sensitivity studies be done before instituting topical, oral or intravenous antibiotics. The bacterial isolates from the ear discharges of CSOM patients in this study present a pattern similar to studies done internationally (including Staphylococcus aureus and Pseudomonas aeruginosa as the most common bacterial isolates), but an increasing rate of resistance of these isolates to the antibiotics commonly used to treat the disease was observed. Majority of isolates of $S$. aureus and pseudomonas were resistant to penicillin in more than $50 \%$ of cases. Macrolides and aminoglycosides were effective against most of the isolates of $S$. aureus and ciprofloxacin was shown to be active against Pseudomonas aeruginosa. These patterns may be useful in instituting the narrowest spectrum systemic antibiotic for CSOM in our setting. If feasible, future studies may include cultures for anaerobes and fungal microorganisms.

\section{REFERENCES:}

1 Acuin J; Department of Child and Adolescent Health Development and Team for Prevention of Blindness and Deafness. Chronic suppurative otitis media: burden of illness and management options. Geneva: World Health Organization 2004

2. Bluestone CD. Epidemiology and pathogenesis of chronic suppurative otitis media: implications for prevention and treatment. Int J Pediatr Otorhinolaryngol 1998; 42:207-223.

3. Gupta V, Gupta A, Sivarajan K. Chronic suppurative otitis media: an aerobic microbiological study. Indian J Otol 1998 Jun;4(2):79-82.

4. Suzuki K, Nishimura T, Baba S, et al. Topical ofloxacin for chronic suppurative otitis media and acute exacerbation of chronic otitis media: optimum duration of treatment. Otol Neurotol. 2003 May;24(3):447-52.

5. Abes G, Espallardo N, Tong M et al. A systematic review of the effectiveness of ofloxacin otic solution for the treatment of suppurative otitis media. ORL J Otorhinolaryngol Relat Spec. 2003 Mar-Apr;65(2):106-16.

6. Wintermeyer SM, Nahata MC. Chronic suppurative otitis media. Ann Pharmacother. 1994 Sep;28(9):1089-99.

7. Kenna MA, Bluestone CD. Microbiology of chronic suppurative otitis media in children. Pediatr Infect Dis J. 1986 Mar-Apr;5(2):223-5.

8. Aslam MA, Ahmed Z, Azim R. Microbiology and drug sensitivity patterns of chronic suppurative otitis media. J Coll Physicians Surg Pak. 2004 Aug;14(8):459-61.

9. Philippine Society of Otolaryngology Head and Neck Surgery, Task Force on Clinical Practice Guideline. Consensus report on chronic suppurative otitis media. 2004 (forthcoming).

10. Pichichero ME. Judicious use of antibiotics in pediatric respiratory infections 2000. Pediatr Infect Dis J. 2000 Sep;19(9):909-10. 